\title{
Practice Spotlight: Pharmacists in a Multidisciplinary Atrial Fibrillation Clinic
}

\author{
Stephen Shalansky, PharmD, ACPR, FCSHP \\ Clinical Coordinator \\ Sonia Basi, BSc(Pharm), ACPR \\ Clinical Pharmacist \\ Christine Yu, BSc(Pharm), ACPR \\ Clinical Pharmacist \\ Pharmacy Department, Providence Healthcare \\ Part of Lower Mainland Pharmacy Services
}

$\mathrm{T}^{\mathrm{k}}$ he St Paul's Hospital Atrial Fibrillation Clinic opened in October 2009 with the mandate of streamlining care for patients with atrial fibrillation who have had suboptimal response to standard therapies and therefore require more complex medication management and/or radiofrequency ablation. The proven benefits of this treatment modality have resulted in broader use and hence long waiting lists for the procedure. It was anticipated that a multidisciplinary approach to care would increase patients' access to clinicians familiar with the various treatment options. Therefore, the clinic employs a registered nurse, a nurse practitioner, and a clinical pharmacist (with Sonia Basi and Christine Yu sharing the clinical pharmacist role), all of whom work closely with a team of electrophysiologists. Each discipline utilizes the full extent of its professional authorities for triaging, selection of treatment, and follow-up. Atrial fibrillation clinics successfully using this multidisciplinary approach at other sites in Canada, the United States, and the United Kingdom have been described in the literature. ${ }^{1-3}$ However, for the St Paul's Hospital Atrial Fibrillation Clinic, the specific roles and activities for each discipline were developed in house, since this was the first such clinic in the province of British Columbia.

Cardiologists make most referrals to the clinic, but patients may also be referred by general internists, family physicians, other electrophysiologists, or emergency physicians. The clinic nurse conducts a brief triage interview by telephone with each patient to assess the severity and urgency of symptoms. All patients with documented atrial fibrillation or flutter are entered into clinic care and scheduled for an hour-long group education session run by the nurse and the clinical pharmacist. Topics covered in the session include the pathophysiology of atrial fibrillation, signs and symptoms, efficacy and toxicity of commonly used medications, and value and role of various treatment procedures, including radiofrequency ablation. This information enables the patient to have a more informed discussion with one of the clinic's electrophysiologists and facilitates collaborative decision-making regarding treatment.

During a patient's initial on-site visit to the clinic, he or she is seen first by a nurse or nurse practitioner, who conducts a physical examination and obtains the medical and medication history. Next, one of the electrophysiologists interviews the patient for consideration of treatment options. If the history suggests a complicated medication history, the clinical pharmacist also participates in this session. Treatment options are discussed with the patient, and a treatment course is chosen. For initiation of new medications or changes to existing regimens, decisions typically represent a collaborative effort involving the pharmacist, the electrophysiologist, and the patient. The pharmacist counsels the patient about any new medications before the clinic visit ends.

Follow-up, conducted primarily by the pharmacist and the nurse practitioner, depends on the treatment course prescribed and the severity of symptoms. About $40 \%$ of the patients seen at the clinic undergo radiofrequency ablation; therefore, much of the pharmacist's time is spent ensuring that the medication regimen is appropriate in the periablation period. All patients who undergo ablation receive anticoagulation, unless it is contraindicated. The pharmacist is responsible for adjusting the dose of warfarin, as well as ordering and interpreting the results of international normalized ratio (INR) tests. Careful dose titration is required, because the clinic electrophysiologists prefer to perform ablation when the INR is between 2.0 and 2.5 (on the basis of published evidence ${ }^{4,5}$ and personal preference). The pharmacist contacts the patient 1 week after the procedure to continue anticoagulation management, and the nurse practitioner follows up 3 months after ablation, consulting the pharmacist if there are any medication-related questions. Many patients are maintained on antiarrhythmic agents in the postablation period, so follow-up often includes discussion about the efficacy of and adverse events associated with these 
agents. Patients are encouraged to contact the clinic if there are any issues related to their atrial fibrillation or their medications, and each patient is seen in person by one of the electrophysiologists after 6 months.

Patients who do not undergo ablation are managed with medications that control heart rate and/or rhythm and may also receive anticoagulants. The frequency of follow-up phone calls and visits varies, depending on the plan prescribed by the electrophysiologist during the first clinic visit. As for ablation patients, follow-up for these patients is conducted primarily by the pharmacist and the nurse practitioner. For patients whose atrial fibrillation medications are changed as a result of their visit to the clinic, the pharmacist will follow up within 4 to 6 weeks to inquire about efficacy and any toxic effects. The pharmacist and the nurse practitioner are also responsible for arranging scheduled monitoring, including determination of serum creatinine and thyroid-stimulating hormone, liver function tests, electrocardiography, pulmonary function tests, chest radiography, and eye examinations, on the basis of a medication-specific schedule developed by the clinic staff.

The clinical pharmacist's position and practice at the St Paul's Hospital Atrial Fibrillation Clinic are unique in several ways. First, the clinic at St Paul's Hospital has a full-time clinical pharmacist position, as opposed to a part-time pharmacist or no pharmacist at all, and this person has a broader scope of responsibility than pharmacists working in other atrial fibrillation clinics described in the literature or operating in British Columbia. ${ }^{1-3}$ Second, the hospital's Pharmacy Department was not involved in preparing the original proposal, yet the budget included the pharmacist position. The hospital's director of cardiology, a coauthor on the initial proposal, had a great deal of positive experience with clinical pharmacy services for inpatient care and felt that a pharmacist would be key to the success of the proposed multidisciplinary outpatient clinic model. Third, the close collaboration among the electrophysiologists, a registered nurse, and a nurse practitioner is unique in British Columbia and sets a standard for future collaborative care models within the province. Fourth, the short-term, narrow therapeutic range for warfarin in the preablation period presents a unique challenge for the titration of anticoagulation. For example, the ablation procedure may need to be rescheduled if anticoagulation is not well managed, which makes dose titration a particularly important responsibility. Finally, Cardiac Services British Columbia has set key outcomes for the purpose of monitoring the success of the atrial fibrillation clinics in the province, and several of these key outcomes focus on the pharmacist's role. These include time in the therapeutic range for patients taking warfarin and number of changes to the medication regimen (either drug or dose). These key outcomes will provide an opportunity for objective evaluation of some of the pharmacist's clinical activities.
With the increased focus on outpatient clinics in health care today, the increased prevalence of atrial fibrillation, and the increasing number of ablation procedures being performed, it is likely that more atrial fibrillation clinics will be opening across Canada in the foreseeable future. In British Columbia, 2 clinics were opened initially (including the one at St Paul's Hospital); their success led to the opening of 2 additional clinics, and a fifth clinic is planned. Thus, there will be opportunities to implement similar positions for clinical pharmacists or to increase the pharmacist's role in existing clinics. Pharmacists and administrators working in settings where patients with atrial fibrillation are commonly treated should be alert for plans to implement atrial fibrillation clinics and get involved early in the planning stages. Patient care models differ between clinics, so site visits can offer valuable insight into the development of care models, logistics, and "clinical pearls". For existing clinics with little or no involvement of pharmacists, proposals to increase the pharmacist's role can be based on the increasing experience and literature relating to this subject. This setting offers an excellent opportunity for clinical pharmacists to practice to the full extent of their clinical knowledge and legislated authority. Success in this role could be used as a precedent for increasing the responsibility of clinical pharmacists working on multidisciplinary teams in other specialty areas.

\section{References}

1. Gillis AM, Burland L, Arnburg B, Kmet C, Pollak T. Treating the right patient at the right time: an innovative approach to the management of atrial fibrillation. Can J Cardiol 2008;24(3):195-198.

2. Martins JL, Fox KF, Wood DA, Lefroy DC, Collier TJ, Peters NS. Rapid access arrhythmia clinic for the diagnosis and management of new arrhythmias presenting in the community: a prospective, descriptive study. Heart 2004;90:877-881.

3. Holding S, Tyndall K, Russell C, Cowan C. The impact of a nurse-led rapid-access atrial fibrillation clinic. Br J Cardiac Nurs 2009;4(6):276-281.

4. Hussein AA, Martin DO, Saliba W, Patel D, Karim S, Batal O, et al. Radiofrequency ablation of atrial fibrillation under therapeutic international normalized ratio: a safe and efficacious periprocedural anticoagulation strategy. Heart Rhythm 2009;6(10):1425-1429.

5. Schmidt M, Segerson NM, Marschang H, Akoum N, Rittger H, Clifford SM, et al. Atrial fibrillation ablation in patients with therapeutic international normalized ratios. Pacing Clin Electrophysiol 2009;32(8):995-999.

The Practice Spotlight series highlights the accomplishments of Canadian pharmacists with unique practices in hospitals and related health care settings. If you have a unique or innovative practice, or you know someone else who should be profiled, please submit your contact information to Mary Ensom, Editor of CJHP (cjhpedit@cshp.ca), and one of our Associate Editors will be in touch with you. 